\title{
Epigenetics and Aging
}

\author{
Thomas A. Rando \\ Department of Neurology and Neurological Sciences, Stanford University School of Medicine, \\ Stanford, CA 94305-5235, USA
}

\begin{abstract}
Epigenetics is the field of study of phenotypic changes that may occur in a cell independent of changes in the underlying genetic code. In the strictest sense, these phenotypic changes are inherited and stable through rounds of DNA replication and cell division. Thus, a skin cell continues to express genes characteristic of that tissue during the proliferation that occurs during normal homeostatic turnover; those cells do not begin to express genes typical of the liver or brain or heart, even though the genomic sequences in the cells of all of those tissues in an individual are identical. In the least strict sense, epigenetic processes are often invoked to explain changes in the regulation of transcription of individual genes even in a non-dividing cell. Given this more inclusive definition of epigenetics, it is no surprise that the profound changes that occur with age in cells and tissues have been hypothesized to be due, either in part or even predominantly, to epigenetic processes. This hypothesis is in contrast to, but not mutually exclusive of, the notion that phenotypic changes with age result from the accumulation of nuclear and mitochondrial DNA mutations. Although the accumulation of DNA mutations can surely lead to the structural and functional decline of a tissue by impairing cellular function, there is little evidence that DNA mutations alone can account for the characteristic changes of aging that are shared across individuals within a species and even across species. As such, the hypothesis that epigenetic changes underlie age-related phenotypic changes of cells and tissue, as well as the age-dependent increase in susceptibility of the organism to many diseases, during aging has gained considerable support.
\end{abstract}

Among the most well characterized and widely studied epigenetic changes are methylation of DNA itself and post-translational modifications of histones. Histones are key components of nucleosomes, the fundamental repeating structures of chromatin composed of DNA wound around histone octamers. There are many post-translational modifications of histones, but the modifications that are primarily studied in the context of epigenetic regulation are acetylation and methylation. These epigenetic marks on DNA and histones, and their corresponding methylases/demethylases and acetylases/deacetylases, help to define the patterns of gene expression that distinguish one cell type from another, genes that are repressed versus those that are expressed in a given cell, and the code that governs which genes are poised to be expressed. What is clear from current lines of research is that there are many epigenetic marks that change with age, and this has led to the notion that the changes may be proximal causes of phenotypic changes of cells and tissues.

In 2007, a workshop sponsored by the National Institute on Aging was held to discuss this topic. The theme workshop, titled "Epigenetics of Aging and Age-Related Diseases", featured presentations and discussions by investigators with interests ranging from yeast genetics to

Publisher's Disclaimer: This is a PDF file of an unedited manuscript that has been accepted for publication. As a service to our customers we are providing this early version of the manuscript. The manuscript will undergo copyediting, typesetting, and review of the resulting proof before it is published in its final citable form. Please note that during the production process errors may be discovered which could affect the content, and all legal disclaimers that apply to the journal pertain. 
Alzheimer's disease. This special issue of Experimental Gerontology contains select papers that were presented at the workshop and offers a sampling of the topics discussed.

Thompson et al. provide a general review of epigenetic changes that occur in cells, mechanisms that regulate those changes, and tools that are currently available to examine the epigenome. They then consider how epigenetic dysregulation may contribute to the aging process. Lamceta and colleagues present the hypothesis that dysregulation of microRNA expression in mid-life, leading to epigenetic imbalances, may underlie the profound age-related changes that occur later in life, including the susceptibility to age-related diseases. Willis-Martinez et al. provide a review of the role of changes in histone acetylation in aging and age-related diseases, focusing specifically on histone deacetylases (HDACs). They examine the specific HDACs in regulating cell proliferation and being a determinant of whether a cell will undergo differentiation, senescence, apoptosis, or oncogenic transformation, highlighting the role of the epigenetic state in the balance between aging and cancer. Ergen and Goodell present studies of changes in the properties and transcriptome of hematopoietic stem cells with age that suggest a link between aging, genomic instability, and epigenetic dysregulation. Lahiri and Maloney discuss a hypothesis that environmentally-induced epigenetic changes, particularly changes in DNA methylation, predispose individuals to the later development of Alzheimer's disease when additional stresses are placed on epigenetically-dysregulated cells. Michels explores the interface between epigenetics and epidemiology, examining both the similarities of techniques used in each field and the value to interdisciplinary research that is specifically focused on epigenetic epidemiology. In this new field, it is the epigenetic state that is considered a key link between environmental exposure and disease risk. This is clearly a field that will attract the interest of gerontologists.

Together, these reports reflect the breadth of topics that were discussed over the two-day workshop. Two additional papers, not discussed at the NIA workshop, have been included in this special issue on epigenetics as they complement the studies discussed above.

Grillari and Grillari-Voglauer examine the role of non-coding RNAs in the regulation of cellular senescence, in the control of gene silencing during aging, and in the modulation of conserved pathways that influence longevity. Li et al examine how age-dependent changes in DNA methylation are mediated by changes both in the expression of enzymes that regulate DNA methylation and in the availability of micronutrients. Studying T cells from humans of different ages, they find that there is an age-dependent increase in sensitivity to adverse effects of poor nutrition, perhaps predisposing to diseases of aging.

The expanding scope of research in epigenetics coupled with the complex processes of aging guarantee that this will be at the forefront of aging research in the coming decade and will likely lead to a deeper understanding of phenotypic changes with age and the mechanisms of increased risk of development of age-related diseases. 